\title{
Effect of Some Biochemical Parameters Such as Antioxidants in placenta of Infected Pregnant Woman with Toxoplasmosis
}

\author{
Muna I. Ibraheem ${ }^{*}$, Layla A. Mustafa $^{2}$, Ahmed Y. Ismail ${ }^{3}$ \\ 1*Office of Agricultural Research, Mosul, Iraq \\ ${ }^{2}$ Department of Chemistry, Collage of Science, Mosul University, Mosul, Iraq \\ ${ }^{3}$ Department of Basic Science, Collage of Nursing, Mosul, Iraq
}

E-mail: ${ }^{1 *}$ Munaismail12@yahoo.com, ${ }^{2}$ laylamustaf@gmail.com, ${ }^{3}$ ahmad_yahya@uomosul.edu.iq

(Received December 30, 2019; Accepted February 09, 2020; Available online September 01, 2020)

DOI: 10.33899/edusj.2020.126095.1037, (c) 2020, College of Education for Pure Science, University of Mosul.

This is an open access article under the CC BY 4.0 license (http://creativecommons.org/licenses/by/4.0/).

\section{Abstract:}

Reregulation of biochemical profile of host as a result of host cells-pathogen interaction may involve in pathogenesis of toxoplasmosis disease. The biochemical parameters in the placenta of Toxoplasma-infected pregnant women and non-infected pregnant women (control group) were investigated The criteria taken into consideration are: Superoxide dismutase (SOD), Peroxidase (Px) , Glutathione- S- transferase (GST), Lactate dehydrogenase (LDH), Aryl esterase (AE) , Acetylcholine esterase (AchE), Vitamin A,C and E As well as placental electrolytes including: Sodium $\left(\mathrm{Na}^{+}\right)$, Potassium $\left(\mathrm{K}^{+}\right)$, Chloride $\left(\mathrm{Cl}^{-}\right)$, Calcium $\left(\mathrm{Ca}^{++}\right)$, Selenium $(\mathrm{Se})$, Cupper $(\mathrm{Cu})$, Zinc $(\mathrm{Zn})$, Iron $(\mathrm{Fe})$, Magnesium $(\mathrm{Mg})$ and Manganese $(\mathrm{Mn})$. The results showed: a nonsignificant increase in the activity of SOD, GST, LDH and AchE during 6-9 months, and activity of $\mathrm{AE}$ during 1-3 months, were determined whereas the activity of Px was decreased in all periods of pregnancyA non- significant increase in the level of vitamin $E$ and non-significant decrease in levels of vitamin $\mathrm{A}$ and $\mathrm{C}$ during pregnancy periods in placentae of infected pregnant Were observed. . A significant increase $(\mathrm{p} \leq 0.01)$ in levels of $\mathrm{Na}^{+}$and $\mathrm{K}^{+}$during 1-3 months and $\mathrm{Ca}^{++}$during 3-6 months, whereas the level of $\mathrm{Cl}^{-}$decreased during pregnancy periods. A significant increase $(\mathrm{p} \leq 0.01)$ in levels of Seand $\mathrm{Mg}$ during 6-9 months and a non- significant increase in level of $\mathrm{Cu}$ during 1-3 months and 6-9 months, whereas the level of $\mathrm{Zn}, \mathrm{Fe}$ and $\mathrm{Mn}$ significantly decreased in all periods of Pregnancy in placenta infected women.

Keywords: Toxoplasmosis, Placenta, Vitamins, Enzymes, Trace elements.

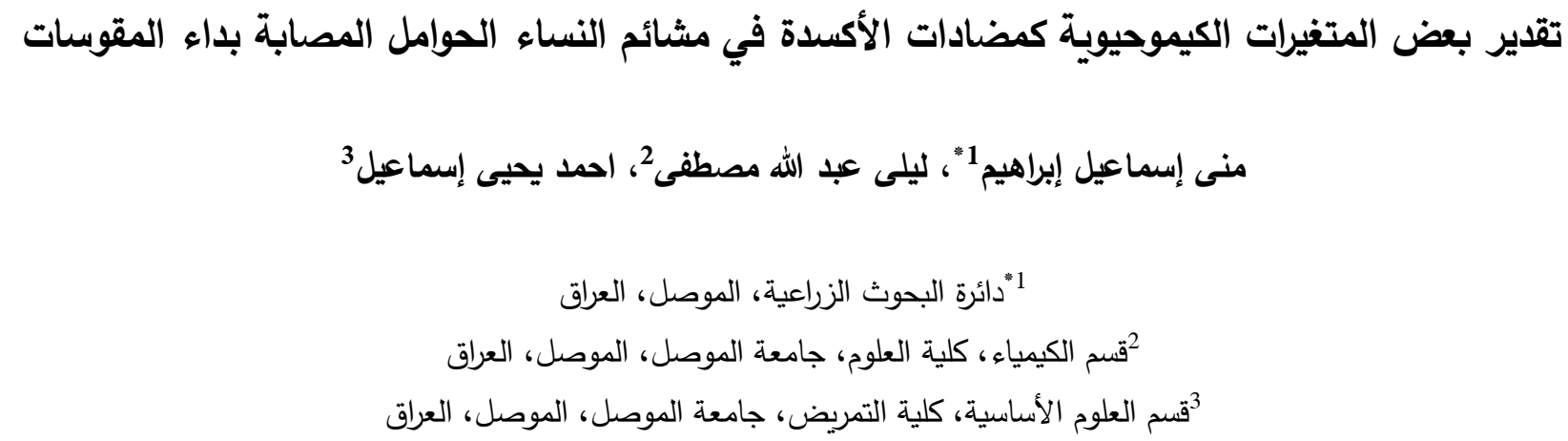


إعادة تتظيم الثاكلة الكيموحيوية للمضيف نتيجة التفاعل بين خلايا المضيف والكائن المرض قد يساهم في إمراضية داء المقوسات. فقد تم قياس السوبرأوكسايد دسميوتيز (SOD) والبيروكسيديز (Px)والكلوتاثيون S- وترانسفيريز (GST) واللاكتيت ديهادروجنيز (LDH) والأريل إستريز (AE) والأسيتايل كولين إستريز (AchE) والفيتامينات C،E،A، بالاضافة التى مستوى الثوارد

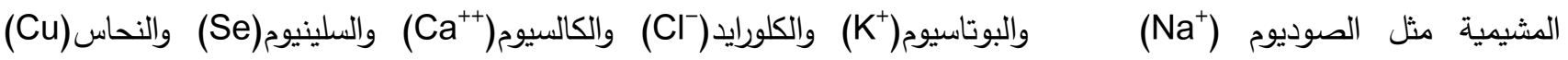
والخارصين(Zn) والحديد (Fe)والمغنيسيوم (Mg) والمنغنيز (Mn). وأظهرت النتائج لمشائم النساء الحوامل المصابة بداء المقوسات

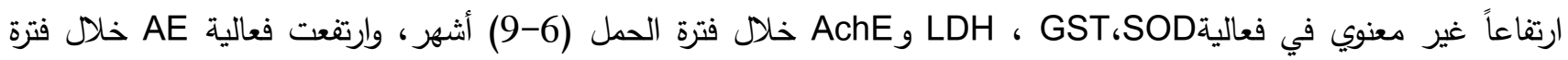
الحمل (1-3) أشهر في حين انخفضت فعالية Px خلال فترات الحمل في مشائم النساء الحوامل المصابة. وأظهرت الفيتامينات ارتفاعاً غير معنوي في مستوى فيتامين E وانخفاضًا غير معنوي في مستوى فيتامين A و خلال فترات الحمل في مشائم النساء

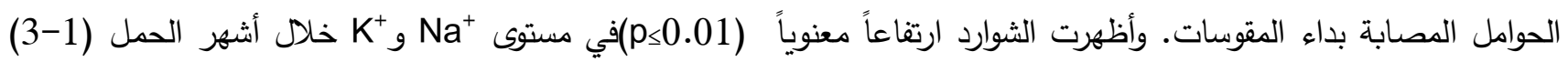

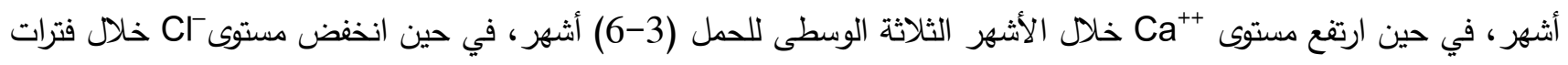

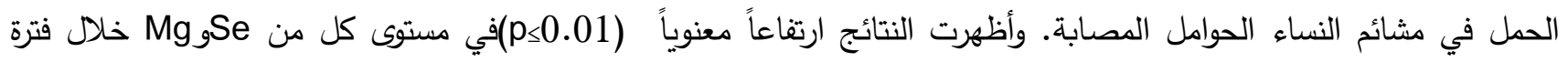

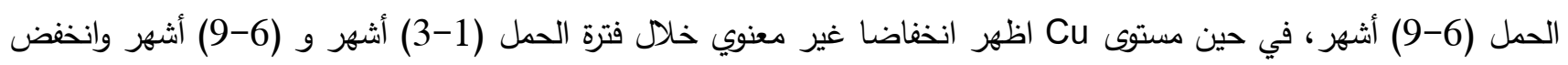
مستوى Zn و Fe ,Zn خلال فترات الحمل في مشائم النساء الحوامل المصابة بداء المقوسات الكوندية. الكلمات الادلة: داء المقوسات، المشيمة، فيتامينات، إنزيمات، عناصر نزره.

المقدمة:

تعتبر المشيمة عضواً بسيط التركيب متعدد الوظائف يتطور خلال مرحلة الحمل، يرافق الجنين في نموه ويوفر للجنين ما

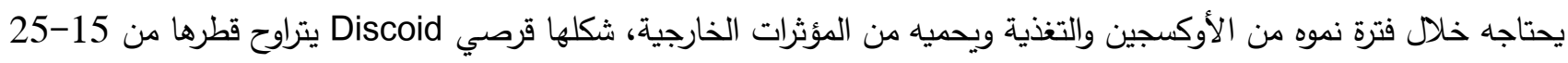

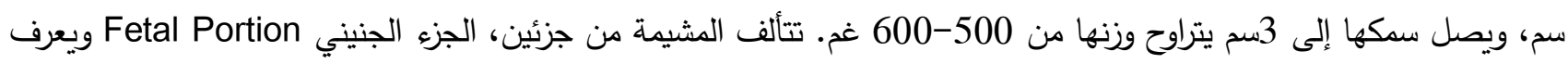

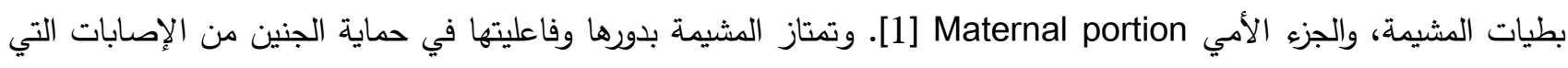

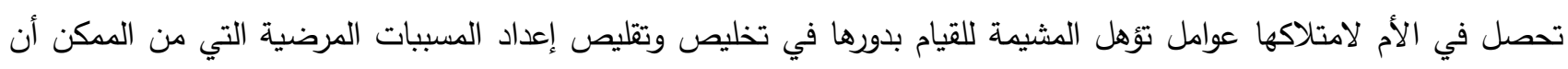

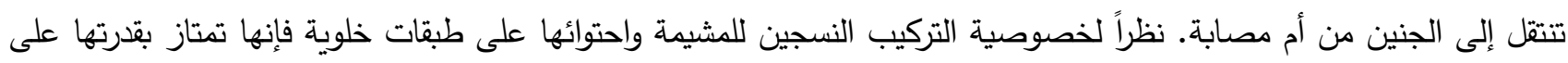

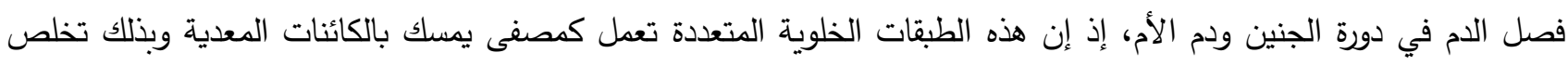
الجنين من وصول هذه الكائنات إليه ومنها طفيل المقوسة الكوندية، إذ إن احتمالية وصول الطفيل إلى الجنين قد يدل يحدث نتيجة التلف الموضعي في إحدى الطبقات النسجية للمشيمة. استخدمت المشيمة في هذه الدراسة بوصفها أحد المعايير المعتمدة والتي من الممكن اعتمادها مؤشراً لكثف الإصابة الخلقية في حال عزل الطفيل منها، في مستخلص المشيمة في النساء المصابات بالمرض (المجهضات وغير المجهضات) من هنا

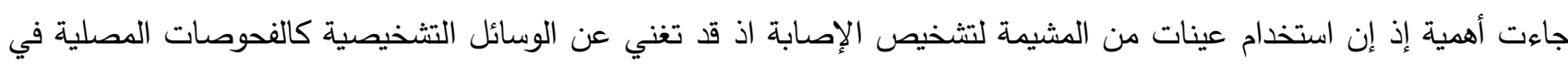

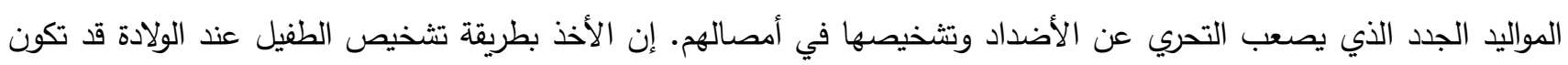
مفيدة وذات أهمية من الناحية الطبية وإحدى الطرائق المهمة للحد من الإصابة بداء المقوسات [2]. تعد هذه الدراسة الاولى لإنى المتضمنة قياس العديد من المتغيرات الكيموحيويه من إنزيمات، فيتامينات ومضادات أكسده لملاحظه تأثير طفيلي داء المقوسات في المشيمه التي تعتبر عضو حماية للجنين خلال فتره الحمل. 


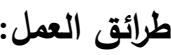
جمع عينات المشيمة:

جمعت عينات المشيمة من نساء مجهضات وغير مجهضات وبعدد 24 عينة من مستثفى الخنساء للنسائية والتوليد. إذ جمعت 18

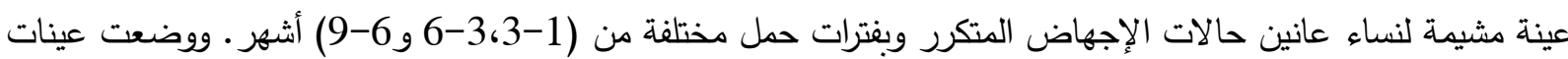
المشيمة في حاويات زجاجية تحتوي محلولاً ملحياً المقوسات بطريقة الفحص المجري المباشر لمسحات من مستخلص المشيمة المصبوغة بصبغة كيمزا. عزل الطفيل: تم عزل الطفيل بصورته النقية من المشائم المخمجة بإتباع الخطوات الآتية:[- 4 - 3] ـ 1- أخذت المشيمة وقطعت إلى قطع صغيرة جداً بواسطة مقص ومشرط حاد ومعقم.

2- سحقت القطع بواسطة هاون خزفي بعد إضافة كمية قليلة من المحلول الملحي الفسلجي NaCl\% 0.9 إليها للحفاظ على حيوية الطفيل. 3- رشح المستخلص باستخدام عدة طبقات من الثاش لغرض التخلص من القطع الكبيرة. 4- أجريت عملية الطرد المركزي للنسيج بسرعة 3000 دورة/ دقيقة لمدة 10 دقائق، بعد ذلك أخذ الراسب وعلق في المحلول

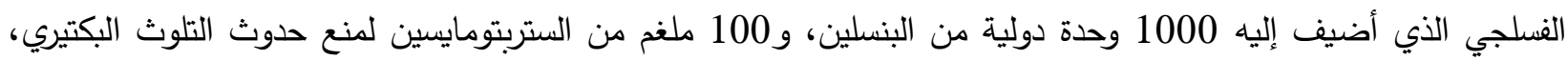
ووضع المعلق في الثلاجة بدرجة 4 م لحين الاستخدام.

قياس المتغيرات الكيموحيوية: تم قياس متغيرات الكيموحيوية في مستخلص مشائم النساء الحوامل المصابة بداء المقوسات والسليمة ومنها: قياس فعالية إنزيم سوبر أوكسايد دسميوتيز :

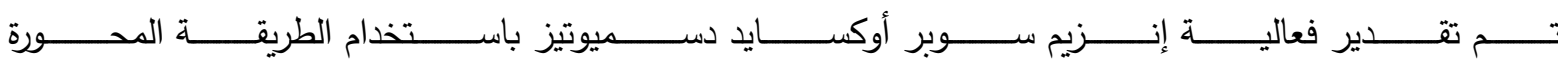

.[6] Modified Riboflavin/Nitro Blue Tetrazolium

$$
\text { قياس فعالية البيروكسيديز }
$$

قدر باستخدم طريقة لونية وذلك باستعمال مادة 4 -أمينوانتيبايرين .amino antipyrine

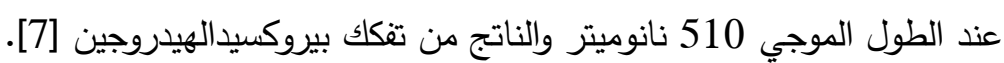
تقدير فعالية إنزيم كلوتاثيون الناقل للكبريت:

حسب الطريقة المذكورة من قبل الباحثين .

تقدير فعالية لاكتيت ديهايدروجينيز.

تم تقدير فعالية إنزيم لاكتيت ديهايدروجينيز باستخدام عدة جاهزة من شركة SRYBo] تقدير فعالية إنزيم أريل إستيريز. قدرت فعاليته حسب طريقة . Tomas et al [10] إذ يعمل الإنزيم على تحليل مادة فينل أستيت إلى فينول وحامض الخليك. تقدير فعالية الأسيتايل كولين إستريز: استخدمت طريقة الأقطاب الكهربائية Electrometric Techniques بالاختلاف في الاس الهيدروجيني لتقدير فعالية الكولين إستريز [11]. 
: 12 - 12

تم تقدير فيتامين A باستخدام طريقة تعتدد على خاصية الفيتامين في امتصاص الضوء عند الطول الموجي 327 نانوميتر في المنطقة فوق البنفجية والفرق في الامتصاص في العينة، قبل الإشعاع بالأشعة فوق البنفسية وبعده يمكن استخدامه في تقدير الفيتامين A [12]

13

تم تقدير فيتامين E باستخدام الطريقة المعتمدة على تفاعلات أكسدة واختزال تسمى Emmeric-Engle Reaction. عملية فصل فيتامين E من المصل يتم بواسطة استخدام مذيب عضوي الزايلين الذي يعمل على فصل فيتامين E والكاروتين من مستخلص مشائم ثم بعد ذلك تقرأ الامتصاصية عند الطول الموجي 460 نانومتر لتقدير الكاروتين ثم يضـاف كلوريد الحديديك

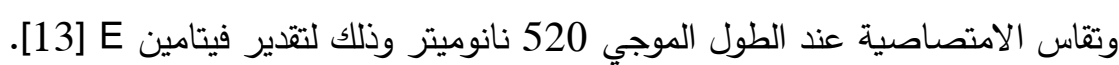

14-تقدير فيتامين

تم تقدير تركيز فيتامين C في مستخلص مشائم باستخدام الطريقة المتبعة من قبل .

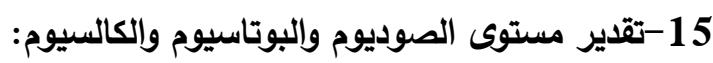

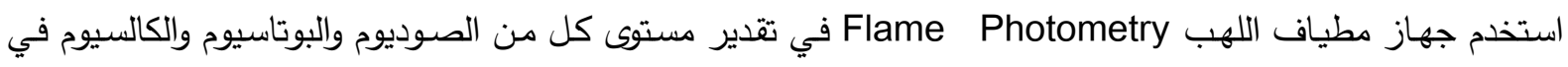
المصل باستخدام مرشح أحادي اللون الذي يمرر الضوء الأصفر فقط العائد لأيون الصوديوم في النموذج عند طول موجي 589 نانوميتر [15]. تقدير مستوى الكلورايد:

استخدمت الطريقة اللونية Colorimetric Method في تقدير مستوى الكلورايد في مستخلص مشائم [9].

العناصر النزرة Trace elements: 16- تقدير تركيز عدد من العناصر النزرة بتقنية طيف الامتصاص الذري

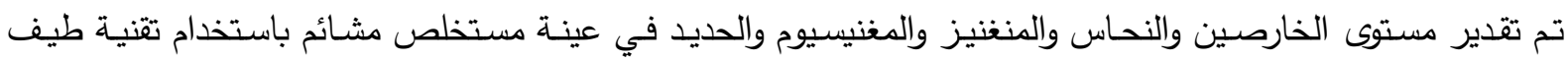
الامتصاص الذري Atomic Absorption Spectrophotometer إذ إنها طريقة حساسة لتقدير أكثر من 60 عنصراً [16]. تقدير تركيز السلينيوم: استخدمت طريقة Allowsh [17 اللونية المحورة في تقدير تركيز السلينيوم ويمتص عند الطول الموجي 335 نانوميتر في الطبقة العضوية.

\section{النتائج والمناقشة:}

تثـير النتائج المشـار اليها في الجدول (1) إلى وجود علاقـة معنويـة عاليـة لفعالية كل من سوبر أوكسايد دسميوتيز وكلوتاثيون - S- وترانسفينز وأريل إستريز في مشائم النساء الحوامل المصابة والسليمات .ولوحظ من الجدول (1) ارتفاع لفعالية سوبر أوكسايد دسميوتيز وكلوتاثيون-S-وترانسفيريز ولاكتيت ديهايدروجينيز و الأسيتايل كولين إستريز في مشائم النساء الحوامل

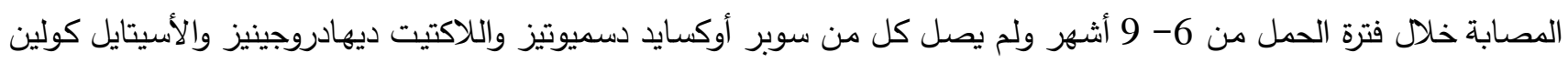

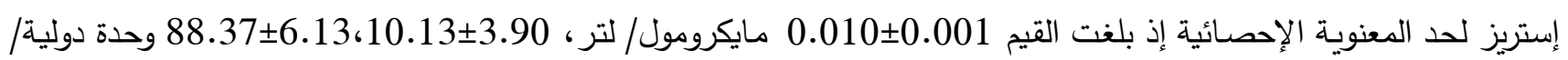
لتر 0.05 0.140 مايكرومول/ لتر،على التوالي. في حين انخفض البيروكسيديز في مشائم النساء الحوامل المصابة خلال فترة

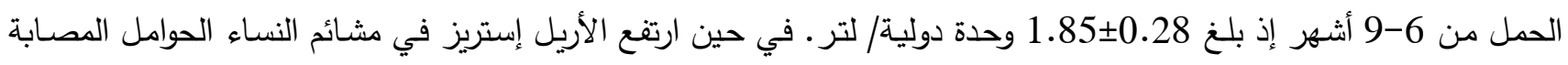

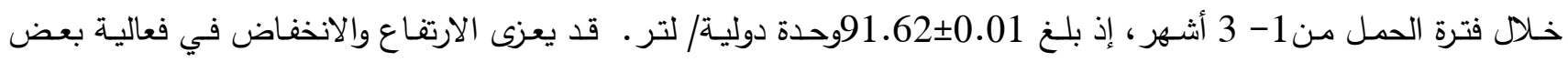
الإنزيمات الى ان سبب زيادة فعالية الإنزيم لتتاقص وغياب المادة الأساس التي يعمل عليها الإنزيم في مناطق النهايات العصبية 
لهذا يتراكم في الدماغ ويظهر بصورة واضحة عند قياس مستواه أو فعاليته في مصل الدم كانزيم الاسيتايل كولين [19-18]. او يعزى السبب إلى تأثير الكرب التأكسدي لاسيما خلال المراحل المتقدمة من الحمل على مضاداد الطات الاكسدة الإنزيمية وتغير تركيب

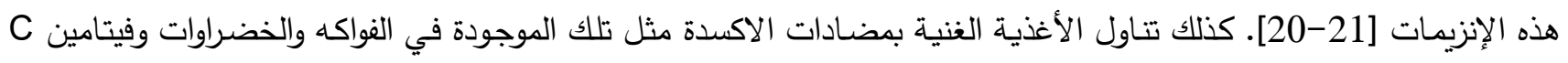

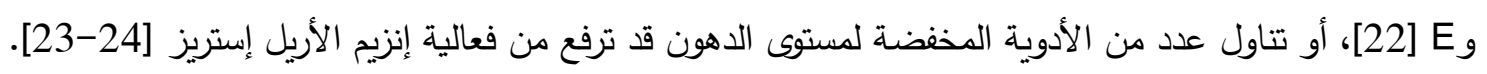
جدول 1 يوضح المعدل SD士 للإنزيمات في مشائم النساء الحوامل المصابة والسليمات.

\begin{tabular}{|c|c|c|c|c|c|c|c|}
\hline $\begin{array}{c}\text { Mean } \pm \\
\text { SD } \mu \mathrm{mol} / \mathrm{L}\end{array}$ & \multicolumn{4}{|c|}{ Mean \pm SDU/L } & $\begin{array}{l}\text { Mean } \pm \\
\text { SD } \mu \mathrm{mol} / \mathrm{L}\end{array}$ & & \\
\hline $\begin{array}{c}\text { أستايل كولين } \\
\text { AchE }\end{array}$ & $\begin{array}{c}\text { أريل إستريز } \\
\text { AE }\end{array}$ & $\begin{array}{c}\text { لاكتيايدروجينيز } \\
\text { LDH }\end{array}$ & $\begin{array}{c}\text { ترانسفيزيز } \\
\text { Sلوتاثيون } \\
\text { GST }\end{array}$ & Px بيروكسيديز & $\begin{array}{c}\text { سوبر أوكسايد } \\
\text { SOD }\end{array}$ & العدد & الحمل المشائم خلال فترات \\
\hline $0.10 \pm 0.05 A$ & $74.62 \pm 9.64 B$ & $80.14 \pm 20.11 A$ & $5.11 \pm 1.80 \mathrm{~B}$ & $18.63 \pm 1.99 \mathrm{~A}$ & $\begin{array}{l}0.008 \pm 0.0 \\
01 \mathrm{~A}\end{array}$ & 6 & مشائم غير المصابة \\
\hline $0.14 \pm 0.01 A$ & $91.62 \pm 0.01 \mathrm{~A}$ & $76.90 \pm 0.01 \mathrm{~A}$ & $7.18 \pm 0.01 B$ & $5.80 \pm 0.01 \mathrm{AB}$ & $\begin{array}{l}0.004 \pm 0.0 \\
01 B\end{array}$ & 6 & مصن(1-3) أشهر \\
\hline $0.10 \pm 0.01 \mathrm{~A}$ & $69.78 \pm 0.01 B$ & $70.42 \pm 2.21 \mathrm{~A}$ & $4.12 \pm 2.22 \mathrm{~B}$ & $3.08 \pm 0.69 B$ & $\begin{array}{l}0.005 \pm 0.0 \\
01 B\end{array}$ & 6 & مصابة من3-6) أثهر \\
\hline $0.14 \pm 0.05 A$ & $73.12 \pm 2.49 \mathrm{~B}$ & $88.37 \pm 6.13 A$ & $10.13 \pm 3.90 \mathrm{~A}$ & $1.85 \pm 0.28 B$ & $\begin{array}{l}0.01 \pm 0.00 \\
1 \mathrm{~A}\end{array}$ & 6 & من مصابة (6-9) أثهر \\
\hline
\end{tabular}

Within columns, means having the same letters don't differ significantly at $\mathrm{p} \leq 0.01$

مستوى الفيتامينات في مشائم النساء الحوامل المصابة والسليمات أوضحت النتائج في لجدول (2) إلى وجود علاقة غير معنوية لمستوى فيتامينA و C في مشائم النساء الحوامل المصابة

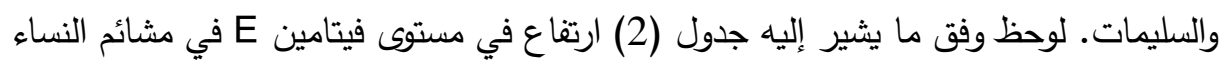

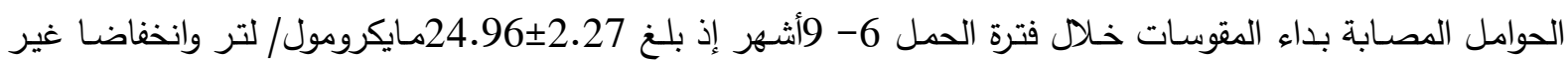

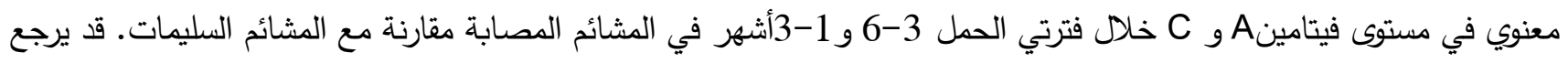

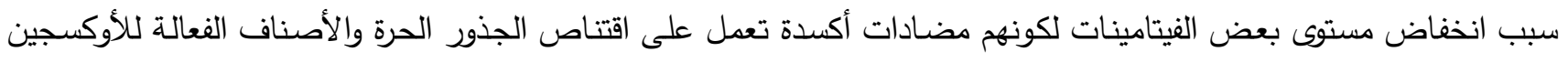

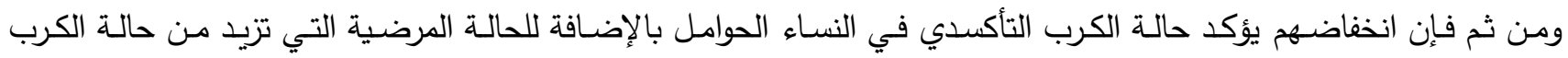
التأكسدي، في حين فيتامين E يعمل على إزالة الاكسدة الناتجة من خلال تثبيط عملية بيروكسيدة الدهن في جدار الخلية ويعمل على حماية الدهون الموجودة فيها من الاكسدة ويقلل من حالة الكرب التأكسدي [25]. 
جدول 2: يوضح المعدل SD士 للفيتامينات في مشائم النساء الحوامل المصابة وإلسليمات.

\begin{tabular}{|c|c|c|c|c|}
\hline \multicolumn{3}{|c|}{ Mean $\pm \mathrm{SD} \mu \mathrm{mol} / \mathrm{L}$} & \multirow[b]{2}{*}{ العدد الع } & \multirow[b]{2}{*}{ المشائم خلال فترة الحمل } \\
\hline فيتامين C & فيتامين E & فيتامين A & & \\
\hline $9.82 \pm 3.52 \mathrm{~A}$ & $24.05 \pm 2.37 A$ & $3.61 \pm 1.90 \mathrm{~B}$ & 6 & مشائم غير المصابة \\
\hline $8.56 \pm 0.01 \mathrm{~A}$ & $21.18 \pm 0.01 \mathrm{~B}$ & $1.47 \pm 0.01 \mathrm{~B}$ & 6 & مصابة من (3-1) أشهر \\
\hline $9.08 \pm 3.777 \mathrm{~A}$ & $24.59 \pm 1.55 \mathrm{~A}$ & $1.26 \pm 0.01 \mathrm{~B}$ & 6 & مصابة من (6-3) أشهر \\
\hline $9.09 \pm 1.42 \mathrm{~A}$ & $24.96 \pm 2.27 \mathrm{~A}$ & $1.54 \pm 1.04 \mathrm{~B}$ & 6 & مصابة من (6-9) أشهر \\
\hline
\end{tabular}

Within columns, means having the same letters don't differ significantly at $\mathrm{p} \leq 0.0$

مستوى الاكتروليتات في مشائم النساء الحوامل المصابة والسليمات أوضحت النتائج في الجدول (3) وجود علاقة معنويـة عالية لمستوى الصـوديوم والبوتاسيوم والكلورايد والكالسيوم لمشائم النساء الحوامل المصابة والسليمات بداء المقوسات. ولوحظ من النتائج الموضحة في الجدول (3) إلى ارتفاع معنوي في تركيز

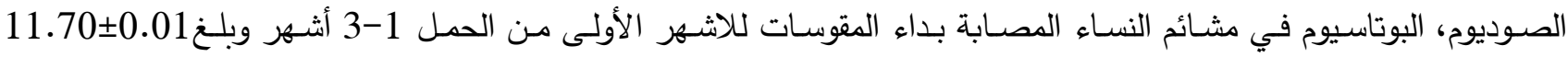
،

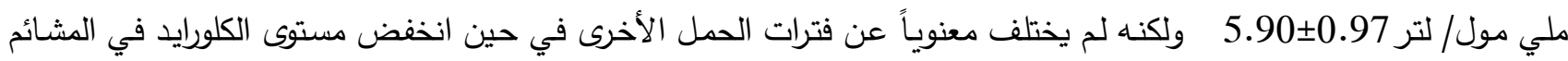

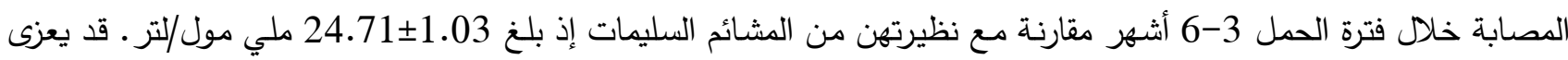

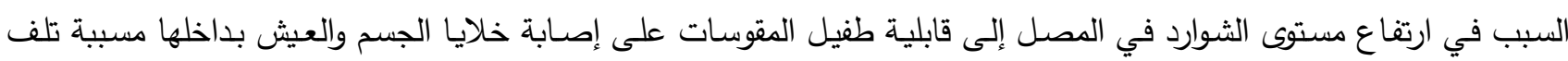

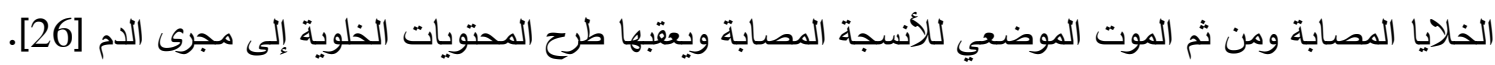

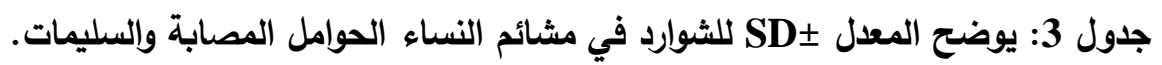

\begin{tabular}{|c|c|c|c|c|c|}
\hline \multicolumn{4}{|c|}{ Mean \pm SD Mmol/L } & \multirow[b]{2}{*}{ العدد } & \multirow[b]{2}{*}{ المشائم خلال فترة الحمل } \\
\hline Ca++ الكالسيوم & الكلوريد Cl- & البوتاسيوم K+ & الصوديوم & & \\
\hline $3.12 \pm 0.68 B$ & $43.96 \pm 15.69 A$ & $36.10 \pm 5.36 \mathrm{C}$ & $5.66 \pm 1.06 \mathrm{C}$ & 6 & مشائم غير المصابة \\
\hline $5.16 \pm 0.01 \mathrm{~A}$ & $35.47 \pm 0.01 \mathrm{AB}$ & $50.23 \pm 0.01 \mathrm{~A}$ & $11.70 \pm 0.01 \mathrm{~A}$ & 6 & مصابة من (1-3) أثنهر \\
\hline $5.90 \pm 0.97 A$ & $24.71 \pm 1.03 B$ & $39.71 \pm 2.79 C$ & $6.50 \pm 1.53 \mathrm{BC}$ & 6 & مصابة من (3-6) أثهر \\
\hline $5.48 \pm 1.33 A$ & $30.33 \pm 6.97 B$ & $45.99 \pm 1.50 \mathrm{~B}$ & $7.35 \pm 0.12 B$ & 6 & مصابة من (6-9) أشهر \\
\hline
\end{tabular}

Within columns, means having the same letters don't differ significantly at $\mathrm{p} \leq 0$. 
مستوى العناصر النزرة في مشائم النساء الحوامل المصابة وإلسليمات

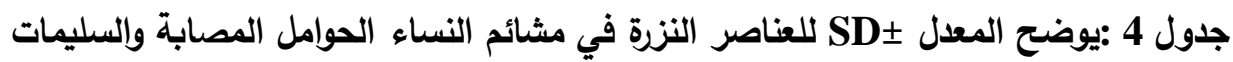

\begin{tabular}{|c|c|c|c|c|c|c|c|}
\hline \multicolumn{6}{|c|}{ Mean \pm SD $\mu \mathrm{mol} / \mathrm{L}$} & \multirow[b]{2}{*}{ العدد العد } & \\
\hline $\begin{array}{l}\text { المنفيز } \\
\text { Mn }\end{array}$ & $\begin{array}{c}\text { المغنيسيوم } \\
\text { Mg }\end{array}$ & الحديد & $\begin{array}{c}\text { الخارصين } \\
\text { Zn }\end{array}$ & النحاس & السلينيوم & & المشائم خلال فترة \\
\hline $0.47 \pm 0.01 \mathrm{~A}$ & $1.00 \pm 0.15 B$ & $1.67 \pm 0.56 \mathrm{~A}$ & $12.01 \pm 6.49 \mathrm{~A}$ & $6.68 \pm 1.70 \mathrm{~A}$ & $0.12 \pm 0.03 C$ & 6 & مشائم غير المصابة \\
\hline $0.23 \pm 0.01 B$ & $0.95 \pm 0.01 B$ & $1.48 \pm 0.01 \mathrm{AB}$ & $10.01 \pm 0.01 \mathrm{~A}$ & $6.02 \pm 0.01 \mathrm{~A}$ & $0.64 \pm 0.01 B$ & 6 & مصابة من (3-1) أشهر \\
\hline $0.23 \pm 0.01 D$ & $0.82 \pm 0.07 C$ & $0.98 \pm 0.01 B$ & $4.66 \pm 1.82 \mathrm{~B}$ & $7.34 \pm 2.70 \mathrm{~A}$ & $0.63 \pm 0.01 B$ & 6 & مصابة من (6-6) أثهر \\
\hline $0.23 \pm 0.01 C$ & $1.14 \pm 0.07 \mathrm{~A}$ & $1.67 \pm 0.74 \mathrm{~A}$ & $3.33 \pm 0.01 B$ & $5.80 \pm 0.88 \mathrm{~A}$ & $0.85 \pm 0.16 A$ & 6 & مصابة من (6-9) أشهر \\
\hline
\end{tabular}

Within columns, means having the same letters don't differ significantly at $\mathrm{p} \leq 0$

أظهرت النتائج في الجدول (4) وجود علاقة معنوية لتركيز السلينيوم والخارصين والمغنيسيوم والمنغنيز في مشائم النساء

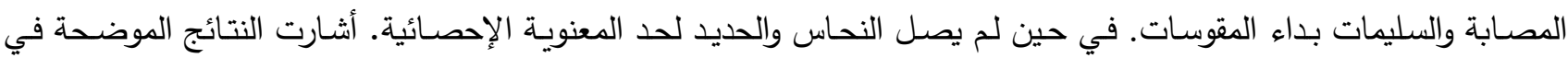
الجدول(4) إلى ارتفاعا في تركيز السلينيوم ، في مشائم النساء الحوامل المصابة بداء المقوسات خلال فترة الحمل من 6- 6- 9 أشهر

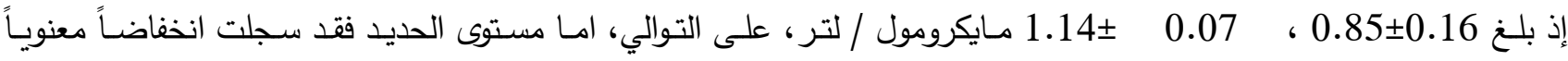
0.98 0.01 في مشائم النساء الحوامل المصابة خلال فترة 3-6 أشهر ، وارتفع النحاس ارتفاعا غير معنوي في المشائم المصابة

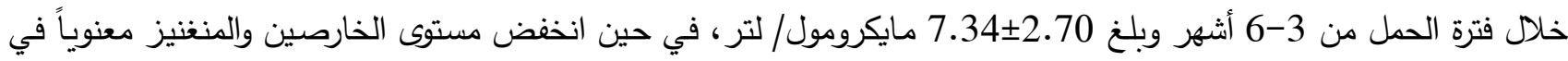

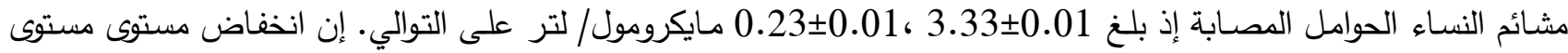

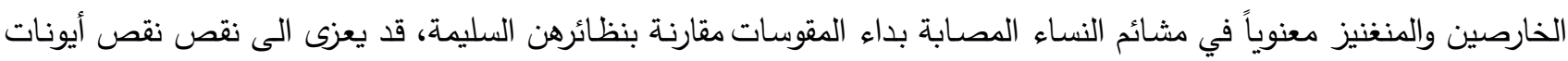
الحديد في النساء الحوامل خلال فترة الحمل إلى استخدام كميات كبيرة خلال بناء الخلايا في الجمم لاسيما كريات الدم الحمر التي لئي

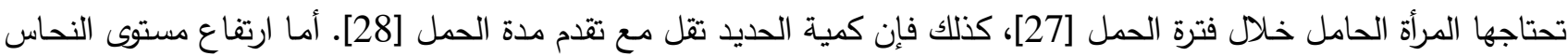
ويعزى إلى وجود اضطرابات بين فعالية أنظمة نقل أيونات الصوديوم ومستوى النحاس في مصل الدم ، إذ إن انخفاض فئل فعالية هذه

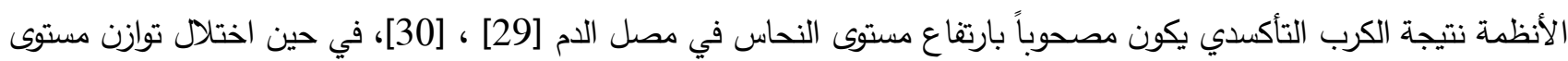

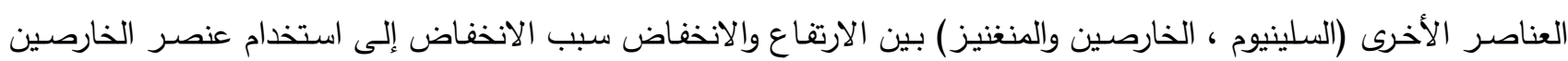
بكميات كبيرة كمرافق لإنزيم سوبر أوكسايد دسميوتيز الذي يبدي فعاليته بوجود العناصر المعدنية (الخارصين، النحاس والمنغنيز) واستخدامه في إزالة جذر السوبر أوكسايد السالب Super oxide anionradical والتخلص منه بتحويله إلى بيروكسيد الهيدروجين وهو من المركبات المؤكسدة غير الحاوية على الجذور الحرة [31]. 
1- Sadler T.W., Langhan's Medical Embryology. $5^{\text {th }}$ ed. Williams and Wrlkins. Company. Bathimore. London. Los Angeles and Sydeny (1985).

2- Remington JS; McLeod R; Thulliez P and Desmants G Toxoplasmosis: In Remington JS; Klein JO Infectious Diseases of the Fetus and New Born Infants". $5^{\text {th }}$ edn. WB Saunders Company, Philadephia,206-346 (2000).

3- Sharma S.P., and Dubey J.P., Ann. J.Vet. Res., 42:128-130 (1981).

4-Dubey JP and Livington JR, Am. J. Vet.,47(3):523-524(1986).

5- AL Khaffaf, F.H.O.Isolation and study of epidemiological seroprevalence of toxoplasmosis age in Nineveh province . M.Sc. Thesis. College of Sciences, University of Mosul. Iraq (2001).

6- Beyer W;Mlay J and Fridovich I ., Res.Mol.Biol.,40:221-253 (1991).

7- Trinder P., Am. Clin. Biochem.,6:24 (1966).

8-Habig W H;Pabst MJ and Jakoby W B, J. Biochem., 249(22):7130-7139 (1974).

9- Tietz NW. Textbook of Clinical Chemistry. $3^{\text {rd }}$ edn.. W.B.Saunders Company, Philadephia,12391250. (1999).

10- Tomas M; Senti M; Gareia- Faria F; Vila J; Torrents A;Govas A and Marrugat J ., Arteriosclar. Thromb. Vasc.Biol.,20:2113. (2000).

11-Mohammad FK and Omer St.V.E.V., Vel.Hum. Toxicol.,24:119-121 (1982).

12- Wotton IDP. Microanalysis in Medical Biochemistry. $6^{\text {th }}$ edn... Edinburge London, 236-237 (1982).

13-Varley H; Gowenlock AH and Bell M.. Vol. (1), London, 222-225,553-555. (1980).

14- Stanley T; David T; Howerds S., Methods in Enzyymology, Vol.62.Vitamin and Coenzymes part D (1979). 
15-Bishop M;Duben J and Fody E .Clinical Chemistry Principle procedure, Correlation, $4^{\text {th }}$ ed.. Lipincott Williams and Wilkins, Philadelphia. (2005).

16-D’Haese PC; Lamberts LV; Vanheule AO and Debroe ME., Clin. Chem.,38(12):2439-2443 (1992).

17-Allowsh TA. Biochemistry study of selenium and its relation with cancer. Ph.D. Thesis,College of Science. Mosul University. (2000).

18-Lancoto, KL ; Herrmann, N. ; Yau, K.K. ; Khan, L.R. ; Liu, B.A. ; Einarson, T.R., Efficacy and safety of cholin esterase inhibitors in Al Zheimers disease: Ameta- analysis. J.Can.Med. Assoc., 196:557-564. (2003).

19- Luchsinger AM., J. Anna. Neural.,33:36-42. (1993).

20-AL-Sabaawy, OM. Measurement of lipid peroxidation irdices and some antioxidations in both blood and sweat fluid of hypertensive patients. M.Sc. Thesis in Biochemistry, College of Sciences, University of Mosul. (2009).

21-Mla-ALw .F. Y.H .Oxidative stress for pregnancy in the Ninawa governorate. M.Sc. Thesis in Biochemistry. College of Sciences, University of Mosul. (2011).

22- Ferre N; Camps J; Fernandez- Ballart J; Arija V; Murphy MM; Ceruelo S; Fidan AF; Cingi CC; Kara fakioglu YS; Vtuk AE; Pekaya S and Piskin FC., J. Animal Vet., 9(12):1707-1711 (2010).

23- Senti M; Tomas M; Fito M; Weinbernner T;Covas M; Sala J; Masia R and Seyrek K; Karagenc T; Pasa S; Kiral F and Atsoy A. Acta.Vet. Brno.,78:471-475 (2009).

24-Dawood M N. Study of liver function tests in non-pregnant women with toxoplasmosis. M.Sc. Thesis, College of Medicine University of Mosul (2007).

25- Mohanty S; Sahu PK; Mandal MK; Mohapatre PC and Panda A., Ind. J. Clin. Biochem.,21(1):101-105. (2006). 
26- Portillo R. Aanemia as the main actor in the infection due to trypanosome evansi. Scitopics. From http:// www. Qa. Scitopics.com (2010).

27-Choi JW and Pai SH., Ann. Clin.Lab.Sci.,30(4):422-428 (2000).

28- Tam KF and Lao TT., J. Paed. Obst. Gyna.,24-28 (2002).

29- Eshratkah B; Sadaghian M; Safari M; Sabri V and Farahmad B., Animal and Veterinary Advances, 7 (4):437-440 (2008).

30- Seyrek K; Karagenc T; Pasa S; Kiral F and Atsoy A., Acta. Vet. Brno.,78:471-475 (2009).

31- Saczko J; Daczweska M; Marcinkowska A; Chwiekowska A; Aaczko Z; Sadler TW Langhan's Medical Emboryology, $5^{\text {th }}$ edn.. Williams and Wrlkins. Company. Bathimore. London. Los Angeles and Sydeny. (1985). 